05,04

\title{
Синтез, структурные и магнитные свойства $\mathrm{NaNiFe}_{2}\left(\mathrm{VO}_{4}\right)_{3}$
}

\author{
(C) Т.В. Дрокина ${ }^{1}$, Д.А. Великанов ${ }^{1}$, О.А. Баюков ${ }^{1}$, М.С. Молокеев ${ }^{1,2}$, Г.А. Петраковский ${ }^{1}$ \\ ${ }^{1}$ Институт физики им. Л.В. Киренского, ФИЦ КНЦ СО РАН, \\ Красноярск, Россия \\ ${ }^{2}$ Сибирский феедеральный университет, \\ Красноярск, Россия \\ E-mail: tvd@iph.krasn.ru
}

Поступила в Редакцию 8 февраля 2021 г.

В окончательной редакции 8 февраля 2021 г.

Принята к публикации 11 февраля 2021 г.

\begin{abstract}
Методом твердофазного синтеза получено новое магнитное соединение $\mathrm{NaNiFe}_{2}\left(\mathrm{VO}_{4}\right)_{3}$, приведены результаты изучения методами рентгеновской дифракции, гамма-резонанса и магнитометрии.

Кристаллическая структура многокомпонентного ванадата описывается триклинной пространственной группой симметрии $P 1$. Элементарная ячейка содержит шесть неэквивалентных смешанных катионных позиций, занятых разновалентными ионами переходных металлов железа $\mathrm{Fe}^{3+}$ и никеля $\mathrm{Ni}^{2+}$, что способствует реализации состояния с локальным нарушением зарядовой нейтральности. Кроме того, обнаружено наличие двух типов позиций атомов железа: с кислородным окружением как в виде октаэдров, так и квадратных пирамид с неравновероятной их заселенностью железом и никелем. Ход температурной и полевой зависимостей намагниченности в широком температурном интервале характерен для парамагнетика, содержащего ферромагнитные кластеры.
\end{abstract}

Ключевые слова: многокомпонентные ванадаты, кристаллическая структура, магнитные свойства.

DOI: $10.21883 /$ FTT.2021.06.50935.020

\section{1. Введение}

Благодаря особенностям кристаллохимии сложных оксидов, содержащих ванадий, предоставляется широкая возможность для синтеза новых соединений с интересными физическими свойствами, открывающих перспективы новых практических применений. Интерес к изучению многокомпонентных ванадатов в значительной степени обусловлен своеобразием кристаллической структуры, допускающей существование конкурирующих магнитных взаимодействий и формирование разнообразных типов магнитного упорядочения [1-5]. В этом аспекте представляет интерес поиск новых соединений на основе ванадия и переходных металлов.

Изучению структурных свойств соединений с общей химической формулой $A \mathrm{CuFe}_{2}\left(\mathrm{VO}_{4}\right)_{3}(A-$ одновалентные щелочноземельные элементы, $A=\mathrm{Na}, \mathrm{Li})$ посвящены работы $[2,4,6,7]$. Из результатов исследований следует, что при комнатной температуре кристаллическая структура $\mathrm{LiCuFe}_{2}\left(\mathrm{VO}_{4}\right)_{3}$ и $\mathrm{NaCuFe}_{2}\left(\mathrm{VO}_{4}\right)_{3}$ описывается триклинной пространственной группой симметрии $P \overline{1}$, элементарная ячейка содержит две неэквивалентные октаэдрические позиции железа Fe1 и $\mathrm{Fe} 2$. Показано, что замещение лития натрием в системе $A \mathrm{CuFe}_{2}\left(\mathrm{VO}_{4}\right)_{3}$ $(A=\mathrm{Na}, \mathrm{Li})$ приводит к трансформации параметров кристаллической решетки, расстояний между магнитными ионами, размера кристаллитов [2].

Магнетизм $\mathrm{LiCuFe}_{2}\left(\mathrm{VO}_{4}\right)_{3}$ характеризуется преимущественно антиферромагнитным обменным взаимодействием и высоким уровнем фрустраций в цепочеч- ной спиновой структуре [4]. В рамках модели косвенной связи оценены параметры обменных взаимодействий в шестиподрешеточном представлении магнетика $\mathrm{LiCuFe}_{2}\left(\mathrm{VO}_{4}\right)_{3}$. Показано, что соединение является антиферромагнетиком с сильными внутрицепочечными и фрустрирующими межцепочечными обменными взаимодействиями [4].

Интересной особенностью свойств соединения $\mathrm{LiCuFe}_{2}\left(\mathrm{VO}_{4}\right)_{3}$, в отличие от $\mathrm{NaCuFe}_{2}\left(\mathrm{VO}_{4}\right)_{3}$, является наличие магнитодиэлектрического эффекта: приложение внешнего магнитного поля приводит к увеличению диэлектрической проницаемости $[8,9]$.

Данные исследования статических магнитных свойств говардэвансита $\mathrm{NaCuFe}_{2}\left(\mathrm{VO}_{4}\right)_{3}$ показали, что наряду с парамагнетизмом и антиферромагнетизмом в данном соединении в области низких температур реализуется неупорядоченное магнитное состояние $[2,8]$. Оценка параметров обменных взаимодействий показала наличие сильных внутрицепочечных и слабых межцепочечных обменных взаимодействий [8].

Проведены исследования резонансных свойств соединений $\mathrm{LiCuFe}_{2}\left(\mathrm{VO}_{4}\right)_{3}$ и $\mathrm{NaCuFe}_{2}\left(\mathrm{VO}_{4}\right)_{3}$, в области температур $110-300 \mathrm{~K}$ в $X$-диапазоне, зафиксирован электронный парамагнитный резонанс, характеризующийся $g$-фактором, равным $2[2,4]$.

Конкуренция обменных взаимодействий разных знака и величины, неоднородное распределение катионов железа по неэквивалентным кристаллографическим позициям приводят, как показали опыты, к зависимости 
магнитных свойств системы $A \mathrm{CuFe}_{2}\left(\mathrm{VO}_{4}\right)_{3}(A=\mathrm{Na}, \mathrm{Li})$ от состава.

Можно ожидать, что введение в структуру сложных ванадатов различных ионов переходных металлов приведет к изменениям кристаллической решетки, способов перекрывания $d$-орбиталей переходных элементов с орбиталями соседних атомов, оказывая влияние на магнитные свойства. Цель настоящей работы заключается в получении нового оксидного материала состава $\mathrm{NaNiFe}_{2}\left(\mathrm{VO}_{4}\right)_{3}$ и экспериментальном изучении его свойств. В настоящей работе представлены результаты измерения структурных и магнитных характеристик сложного ванадата, содержащего ионы переходных элементов.

\section{2. Синтез образцов и техника эксперимента}

Новое соединение $\mathrm{NaNiFe}_{2}\left(\mathrm{VO}_{4}\right)_{3}$ получено реакцией в твердой фазе из смеси окислов высокой чистоты $\mathrm{Na}_{2} \mathrm{CO}_{3}, \mathrm{Fe}_{2} \mathrm{O}_{3}, \mathrm{NiO}, \mathrm{V}_{2} \mathrm{O}_{5}$, взятых в стехиометрическом соотношении

$$
\begin{aligned}
1 / 2\left(\mathrm{Na}_{2} \mathrm{CO}_{3}\right) & +\mathrm{Fe}_{2} \mathrm{O}_{3} \\
& +\mathrm{NiO}+3 / 2\left(\mathrm{~V}_{2} \mathrm{O}_{5}\right) \rightarrow \mathrm{NaNiFe}_{2}\left(\mathrm{VO}_{4}\right)_{3}
\end{aligned}
$$

Образцы в виде спрессованных из смеси реагентов таблеток диаметром $10 \mathrm{~mm}$ и толщиной $1.5-2.0 \mathrm{~mm}$ подвергались высокотемпературной обработке при температурах $600-640^{\circ} \mathrm{C}$ на воздухе. Химический и фазовый состав синтезированного соединения контролировался методом рентгеноструктурного анализа.

Порошковая рентгенограмма $\mathrm{NaNiFe}_{2}\left(\mathrm{VO}_{4}\right)_{3}$ получена при комнатной температуре на дифрактометpe D8 ADVANCE фирмы Bruker, при использовании линейного детектора VANTEC и $\mathrm{Cu} K_{\alpha}$-излучения. Уточнение Ритвельда реализовано при помощи программы TOPAS 4.2 [10]. В ходе рентгеновского эксперимента использовались разные размеры щелей первичного пучка: $0.6 \mathrm{~mm}$ в диапазоне углов $2 \theta=5-70^{\circ}$ и $2 \mathrm{~mm}$ в диапазоне $70-120^{\circ}$. Шаг сканирования равнялся $0.016^{\circ}$ и оставался постоянным на всех участках, время экспозиции на каждом шаге составляло $3 \mathrm{~s}$. Впоследствии вычислены стандартные отклонения интенсивностей всех точек рентгенограммы, а затем интенсивности и стандартные отклонения всех точек высокоугловой части были умножены на нормирующий коэффициент 0.6.

Мёссбауэровские спектры измерены на спектрометре МС-1104Ем (НИИ физики ЮФУ) в геометрии пропускания с радиоактивным источником $\mathrm{Co}^{57}(\mathrm{Rh})$ при комнатной температуре на порошковом образце с составом $5-10 \mathrm{mg} / \mathrm{cm}^{2}$ по естественному содержанию железа.

Статические магнитные характеристики образцов измерены на магнитометрах оригинальной конструкции Института физики им. Л.В. Киренского ФИЦ КНЦ СО РАН [11,12]: СКВИД-магнитометре в интервале температур 4-300 K в магнитном поле $H=0-2 \mathrm{kOe}$, а также на автоматизированном вибрационном магнитометре в интервале температур 77-830 K в магнитном поле до $25 \mathrm{kOe}$. Температурная зависимость магнитного момента измерена в магнитном поле $H=0,1000,2000$ и 2200 Ое. Петли гистерезиса получены при температуpax $T=830,77 \mathrm{~K}$ и комнатной температуре.

\section{3. Экспериментальные результаты и их обсуждение}

\section{1. Структурные свойства $\mathrm{NaNiFe}_{2}\left(\mathrm{VO}_{4}\right)_{3}$}

На рис. 1 показана порошковая рентгенограмма синтезированного соединения $\mathrm{NaNiFe}_{2}\left(\mathrm{VO}_{4}\right)_{3}$, полученная при комнатной температуре.

Практически все рефлексы рентгенограммы образца были проиндицированы триклинной ячейкой с параметрами решетки $a=6.7559$ (1) $\AA, b=8.1309$ (2) $\AA$, $c=9.8098(2) \AA, \quad \alpha=106.346(2)^{\circ}, \quad \beta=104.300(2)^{\circ}$, $\gamma=102.143(2)^{\circ}, V=477.83(2) \AA^{3}$, близкими к таковым y $\mathrm{NaFe}_{3}\left(\mathrm{VO}_{4}\right)_{3}: a=6.757(2) \AA, \quad b=8.155(2) \AA, c=$ $=9.816(3) \AA, \quad \alpha=106.05(2)^{\circ}, \quad \beta=104.401(9)^{\circ}$, $\gamma=102.09(2)^{\circ}, V=480.46 \AA^{3}[13]$, поэтому именно структура $\mathrm{NaFe}_{3}\left(\mathrm{VO}_{4}\right)_{3}$ была использована в качестве исходной. Поскольку уточнение заселенности позиций $\mathrm{Fe} / \mathrm{Ni}$ шло крайне нестабильно из-за близости функций атомного рассеяния, все шесть позиций катионов $\mathrm{Fe}$ были заселены катионами $\mathrm{Fe} / \mathrm{Ni} \mathrm{c}$ фиксированными заселенностями позиций согласно химической формуле $\mathrm{Fe} / \mathrm{Ni}=2: 1$. Координаты $\mathrm{Na} 1$ не уточнялись, что требуется для фиксации начала координат в полярной группе $P 1$. Основные кристаллографические характеристики $\mathrm{NaNiFe}_{2}\left(\mathrm{VO}_{4}\right)_{3}$ и параметры рентгеновского эксперимента приведены в табл. 1.

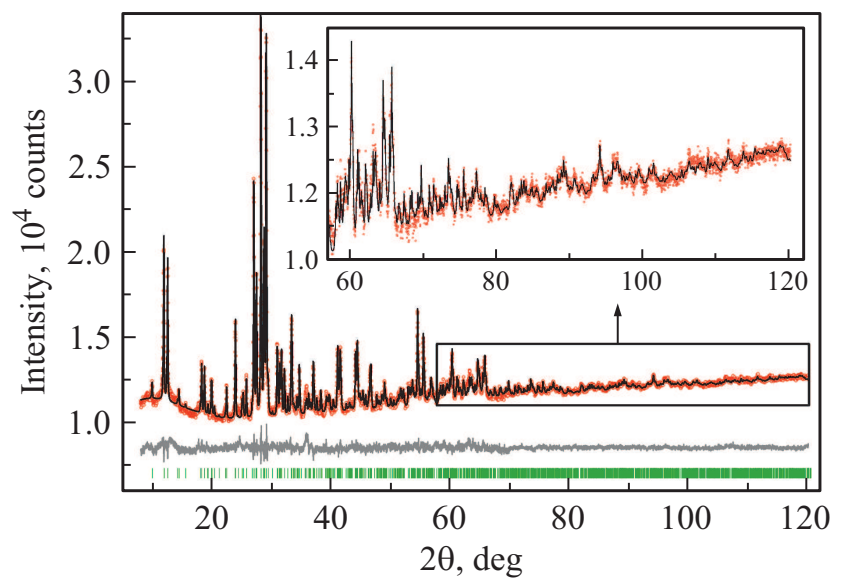

Рис. 1. Рентгенограмма поликристаллического соединения $\mathrm{NaNiFe}_{2}\left(\mathrm{VO}_{4}\right)_{3}$ при комнатной температуре. Разностная рентгенограмма - нижняя кривая. 
Таблица 1. Основные параметры рентгеновского эксперимента, проведенного при температуре $T=300 \mathrm{~K}$, и результаты уточнения кристаллической структуры $\mathrm{NaNiFe}_{2}\left(\mathrm{VO}_{4}\right)_{3}$

\begin{tabular}{c|c}
\hline Пр. гр. & $P_{1}$ \\
\hline$a, \AA$ & $6.7559(1)$ \\
$b, \AA$ & $8.1309(2)$ \\
$c, \AA$ & $9.8098(2)$ \\
$\alpha^{\circ}$, & $106.346(2)$ \\
$\beta^{\circ}$, & $104.300(2)$ \\
$\gamma^{\circ}$, & $102.143(2)$ \\
$V, \AA^{3}$ & $477.83(2)$ \\
$2 \theta$ интервал, & $5-120$ \\
Число рефлексов & 1473 \\
Число уточн. парам. & 160 \\
$R_{w p}, \%$ & 0.97 \\
$R_{p}, \%$ & 0.87 \\
$R_{B}, \%$ & 0.42 \\
$\chi^{2}$ & 1.50
\end{tabular}

Примечание. $a, b, c, \alpha, \beta, \gamma$ - параметры элементарной ячейки; $V$ - объем ячейки; факторы недостоверности: $R_{w p}-$ весовой профильный, $R_{p}$ - профильный, $R_{B}-$ интегральный; $\chi^{2}-$ качество подгонки.

Кристаллическая структура кислородного соединения $\mathrm{NaNiFe}_{2}\left(\mathrm{VO}_{4}\right)_{3}$ описывается триклинной пространственной группой симметрии $P 1$, отличной от $P \overline{1}$, характеризующей ванадаты $\mathrm{LiCuFe}_{2}\left(\mathrm{VO}_{4}\right)_{3}$ и $\mathrm{NaCuFe}_{2}\left(\mathrm{VO}_{4}\right)_{3}$. $\mathrm{B}$ элементарной ячейке исследуемого соединения содержится две формульные единицы $(Z=2)$. Независимая часть ячейки в $\mathrm{NaNiFe}_{2}\left(\mathrm{VO}_{4}\right)_{3}$ (P1) совпадает с элементарной, в отличие от структур в группе $P \overline{1}$, где независимая часть ячейки в 2 раза меньше и число независимых атомов также в 2 раза меньше. Именно наличие/отсутствие центра инверсии приводит к таким отличиям. В остальном эти структуры принципиально не отличаются.

Координаты атомов, заселенность позиций в кристаллической структуре $\mathrm{NaNiFe}_{2}\left(\mathrm{VO}_{4}\right)_{3}$ и тепловые параметры эксперимента представлены в табл. 2, а основные длины связей в кристаллической решетке показаны в табл. 3. Зарядовый состав соединения имеет вид $\mathrm{Na}^{+} \mathrm{Ni}^{2+} \mathrm{Fe}_{2}^{3+}\left(\mathrm{V}^{5+} \mathrm{O}_{4}\right)_{3}$.

Согласно рентгеноструктурным исследованиям, катионы железа $\mathrm{Fe}^{3+}$ в элементарной кристаллической ячейке $\mathrm{NaNiFe}_{2}\left(\mathrm{VO}_{4}\right)_{3}$ занимают шесть кристаллографически неэквивалентных позиций, заселенных совместно с катионами никеля $\mathrm{Ni}^{2+}$ (табл. 2). Четыре из этих шести позиций $\mathrm{Fe} / \mathrm{Ni}$ имеют по кислороду координацию 6 (октаэдр) и две позиции имеют координацию 5 (тригольная бипирамида). Таким образом, одной из особенностей кристаллической структуры исследуемого соединения является локализация катионов разных сорта и валентности в одной и той же позиции кристалла. Это приводит к тому, что в зависимости от соотношения ионов железа и никеля в одной кристаллографической позиции образуется избыток или недостаток положительного заряда, то есть реализуется состояние локального нарушения зарядовой нейтральности.

Для получения дополнительной информации о структуре и характеризации состояния железа в веществе проведено изучение образца $\mathrm{NaNiFe}_{2}\left(\mathrm{VO}_{4}\right)_{3}$ с помощью эффекта Мессбауэра. На рис. 2, а представлен мессбауэровский спектр поликристалла $\mathrm{NaNiFe}_{2}\left(\mathrm{VO}_{4}\right)_{3}$ при комнатной температуре. Обработка спектра выполнена

Таблица 2. Координаты атомов, изотропные тепловые параметры $B_{\text {iso }}\left(\AA^{2}\right)$ и заселенность позиций (Осc.) в кристаллической структуре соединения $\mathrm{NaNiFe}_{2}\left(\mathrm{VO}_{4}\right)_{3}$ при температуре $T=300 \mathrm{~K}$

\begin{tabular}{|c|c|c|c|c|c|}
\hline Атом & $\mathrm{x} / \mathrm{a}$ & $y / b$ & $\mathrm{z} / \mathrm{c}$ & Biso & Occ. \\
\hline $\mathrm{Na} 1$ & 0.99425 & 0.07762 & 0.5534 & $3(1)$ & 1 \\
\hline $\mathrm{Na} 2$ & $0.462(9)$ & $1.018(7)$ & $0.492(7)$ & $0.5(7)$ & 1 \\
\hline $\mathrm{Fe} 1$ & $0.111(7)$ & $0.551(5)$ & $0.400(5)$ & $0.3(2)$ & 0.667 \\
\hline Ni1 & $0.111(7)$ & $0.551(5)$ & $0.400(5)$ & $0.3(2)$ & 0.333 \\
\hline $\mathrm{Fe} 2$ & $0.879(7)$ & $0.448(5)$ & $0.610(5)$ & $0.3(2)$ & 0.667 \\
\hline $\mathrm{Ni} 2$ & $0.879(7)$ & $0.448(5)$ & $0.610(5)$ & $0.3(2)$ & 0.333 \\
\hline $\mathrm{Fe} 3$ & $0.458(7)$ & $0.318(5)$ & $0.984(5)$ & $0.3(2)$ & 0.667 \\
\hline $\mathrm{Ni3}$ & $0.458(7)$ & $0.318(5)$ & $0.984(5)$ & $0.3(2)$ & 0.333 \\
\hline $\mathrm{Fe} 4$ & $0.546(7)$ & $0.733(4)$ & $0.021(5)$ & $0.3(2)$ & 0.667 \\
\hline $\mathrm{Ni} 4$ & $0.546(7)$ & $0.733(4)$ & $0.021(5)$ & $0.3(2)$ & 0.333 \\
\hline $\mathrm{Fe} 5$ & $0.796(7)$ & 0.767 (4) & $0.784(5)$ & $0.3(2)$ & 0.667 \\
\hline Ni5 & $0.796(7)$ & $0.767(4)$ & $0.784(5)$ & $0.3(2)$ & 0.333 \\
\hline Fe6 & $0.234(7)$ & $0.222(5)$ & $0.192(5)$ & $0.3(2)$ & 0.667 \\
\hline Ni6 & $0.234(7)$ & $0.222(5)$ & $0.192(5)$ & $0.3(2)$ & 0.333 \\
\hline V1 & $0.392(8)$ & $0.419(5)$ & $0.664(6)$ & $0.5(2)$ & 1 \\
\hline V2 & $0.606(8)$ & $0.594(5)$ & $0.329(5)$ & $0.5(2)$ & 1 \\
\hline V3 & $0.280(7)$ & $0.839(5)$ & $0.732(5)$ & $0.5(2)$ & 1 \\
\hline V4 & $0.730(7)$ & $0.168(5)$ & $0.261(5)$ & $0.5(2)$ & 1 \\
\hline V5 & $0.090(7)$ & $0.777(5)$ & $0.131(5)$ & $0.5(2)$ & 1 \\
\hline V6 & $0.902(7)$ & $0.216(5)$ & $0.879(5)$ & $0.5(2)$ & 1 \\
\hline O1 & 0.427 (17) & $0.442(11)$ & $0.864(11)$ & $0.5(2)$ & 1 \\
\hline $\mathrm{O} 2$ & $0.577(17)$ & $0.555(11)$ & $0.139(11)$ & $0.5(2)$ & 1 \\
\hline $\mathrm{O} 3$ & $0.366(17)$ & $0.487(11)$ & $0.344(11)$ & $0.5(2)$ & 1 \\
\hline O4 & $0.629(16)$ & $0.537(11)$ & $0.649(11)$ & $0.5(2)$ & 1 \\
\hline O5 & $0.268(13)$ & $0.80(1)$ & $0.56(1)$ & $0.5(2)$ & 1 \\
\hline O6 & $0.743(14)$ & $0.195(11)$ & $0.46(1)$ & $0.5(2)$ & 1 \\
\hline $\mathrm{O} 7$ & $0.500(17)$ & $0.22(1)$ & $0.16(1)$ & $0.5(2)$ & 1 \\
\hline O8 & $0.498(16)$ & 0.76 (1) & $0.828(11)$ & $0.5(2)$ & 1 \\
\hline O9 & $0.149(16)$ & $0.26(1)$ & 0.009 (11) & $0.5(2)$ & 1 \\
\hline O10 & $0.843(16)$ & $0.73(1)$ & $0.994(11)$ & $0.5(2)$ & 1 \\
\hline O11 & $0.180(14)$ & $0.46(1)$ & $0.58(1)$ & $0.5(2)$ & 1 \\
\hline O12 & $0.838(14)$ & $0.51(1)$ & $0.41(1)$ & $0.5(2)$ & 1 \\
\hline O13 & $0.019(17)$ & $0.721(12)$ & $0.739(12)$ & $0.5(2)$ & 1 \\
\hline O14 & 0.977 (17) & $0.286(11)$ & $0.261(11)$ & $0.5(2)$ & 1 \\
\hline O15 & $0.319(17)$ & $0.054(12)$ & $0.824(11)$ & $0.5(2)$ & 1 \\
\hline O16 & $0.682(17)$ & $0.959(12)$ & 0.177 (11) & $0.5(2)$ & 1 \\
\hline O17 & $0.343(15)$ & $0.193(11)$ & $0.58(1)$ & $0.5(2)$ & 1 \\
\hline O18 & $0.666(17)$ & $0.791(11)$ & $0.42(1)$ & $0.5(2)$ & 1 \\
\hline O19 & 0.187 (17) & $0.012(11)$ & $0.24(1)$ & $0.5(2)$ & 1 \\
\hline $\mathrm{O} 20$ & $0.82(2)$ & 0.999 (11) & 0.78 (1) & $0.5(2)$ & 1 \\
\hline $\mathrm{O} 21$ & $0.282(15)$ & $0.734(11)$ & $0.04(1)$ & $0.5(2)$ & 1 \\
\hline $\mathrm{O} 22$ & $0.734(15)$ & $0.270(11)$ & $0.969(11)$ & $0.5(2)$ & 1 \\
\hline $\mathrm{O} 23$ & $0.066(15)$ & $0.655(11)$ & $0.232(8)$ & $0.5(2)$ & 1 \\
\hline $\mathrm{O} 24$ & $0.926(15)$ & $0.332(11)$ & $0.746(9)$ & $0.5(2)$ & 1 \\
\hline
\end{tabular}


Таблица 3. Основные длины связей $(\AA)$ в кристаллической структуре $\mathrm{NaNiFe}_{2}\left(\mathrm{VO}_{4}\right)_{3}$ : (i) $x+1, y, z$; (ii) $x, y+1, z$; (iii) $x-1, y, z$; (iv) $x, y, z+1$; (v) $x, y, z-1$; (vi) $x, y-1, z$; (vii) $x-1, y, z-1$; (viii) $x+1, y, z+1$

\begin{tabular}{|c|c|c|c|}
\hline $\mathrm{Na} 1-\mathrm{O} 6$ & $2.26(6)$ & $\mathrm{Ni} 4-\mathrm{O}^{\mathrm{v}}$ & $1.9(1)$ \\
\hline $\mathrm{Na} 1-\mathrm{O} 17^{\mathrm{i}}$ & $2.27(6)$ & $\mathrm{Ni} 4-\mathrm{O} 10^{\mathrm{V}}$ & $2.09(7)$ \\
\hline $\mathrm{Na} 2-\mathrm{O}^{\mathrm{ii}}$ & $2.29(6)$ & Ni4-O16 & $1.89(9)$ \\
\hline $\mathrm{Na} 2-\mathrm{O} 17^{\mathrm{ii}}$ & $1.88(7)$ & $\mathrm{Ni4}-\mathrm{O} 21$ & $1.85(7)$ \\
\hline $\mathrm{Fe} 1-\mathrm{O} 3$ & $2.05(8)$ & $\mathrm{Fe} 5-\mathrm{O} 4$ & $1.87(8)$ \\
\hline $\mathrm{Fe} 1-\mathrm{O} 5$ & $2.07(8)$ & $\mathrm{Fe} 5-\mathrm{O} 8$ & $2.15(7)$ \\
\hline $\mathrm{Fe} 1-\mathrm{O}^{11}$ & $2.04(8)$ & $\mathrm{Fe} 5-\mathrm{O} 10$ & $2.1(1)$ \\
\hline $\mathrm{Fe} 1-\mathrm{O} 12^{\mathrm{iii}}$ & $1.85(6)$ & $\mathrm{Fe} 5-\mathrm{O} 13^{\mathrm{i}}$ & $1.76(7)$ \\
\hline $\mathrm{Fe} 1-\mathrm{O} 14^{\mathrm{iii}}$ & $2.06(8)$ & $\mathrm{Fe} 5-\mathrm{O} 20$ & $1.89(6)$ \\
\hline $\mathrm{Fe} 1-\mathrm{O} 23$ & $2.04(8)$ & Ni5-O4 & $1.87(8)$ \\
\hline $\mathrm{Ni1}-\mathrm{O} 3$ & $2.05(8)$ & $\mathrm{Ni5}-\mathrm{O} 8$ & $2.15(7)$ \\
\hline Ni1-O5 & $2.07(8)$ & $\mathrm{Ni5}-\mathrm{O} 10$ & $2.1(1)$ \\
\hline $\mathrm{Ni1}-\mathrm{O} 11$ & $2.04(8)$ & $\mathrm{Ni5}-\mathrm{O} 13^{\mathrm{i}}$ & $1.76(7)$ \\
\hline $\mathrm{Ni} 1-\mathrm{O} 12^{\mathrm{iii}}$ & $1.85(6)$ & $\mathrm{Ni} 5-\mathrm{O} 20$ & $1.89(6)$ \\
\hline $\mathrm{Ni1}-\mathrm{O} 14^{\mathrm{iii}}$ & $2.06(8)$ & $\mathrm{Fe} 6-\mathrm{O} 3$ & $2.10(8)$ \\
\hline $\mathrm{Ni1}-\mathrm{O} 23$ & $2.04(8)$ & $\mathrm{Fe} 6-\mathrm{O} 7$ & $1.88(8)$ \\
\hline $\mathrm{Fe} 2-\mathrm{O} 4$ & $2.05(7)$ & $\mathrm{Fe} 6-\mathrm{O} 9$ & $1.88(10)$ \\
\hline $\mathrm{Fe} 2-\mathrm{O} 6$ & $2.03(8)$ & $\mathrm{Fe} 6-\mathrm{O} 14^{\mathrm{iii}}$ & $2.13(8)$ \\
\hline $\mathrm{Fe} 2-\mathrm{O} 11^{\mathrm{i}}$ & $2.13(6)$ & Fe6-O19 & $1.87(6)$ \\
\hline $\mathrm{Fe} 2-\mathrm{O} 12$ & $2.10(9)$ & $\mathrm{Ni6}-\mathrm{O} 3$ & $2.10(8)$ \\
\hline $\mathrm{Fe} 2-\mathrm{O} 13^{\mathrm{i}}$ & $2.09(8)$ & Ni6-O7 & $1.88(8)$ \\
\hline $\mathrm{Fe} 2-\mathrm{O} 24$ & $1.85(8)$ & Ni6-O9 & $1.88(10)$ \\
\hline $\mathrm{Ni2}-\mathrm{O} 4$ & $2.05(7)$ & $\mathrm{Ni6}-\mathrm{O} 14^{\mathrm{iii}}$ & $2.13(8)$ \\
\hline $\mathrm{Ni2}-\mathrm{O} 6$ & $2.03(8)$ & Ni6-O19 & $1.87(6)$ \\
\hline $\mathrm{Ni2}-\mathrm{O} 11^{\mathrm{i}}$ & $2.13(6)$ & $\mathrm{V} 1-\mathrm{O} 1$ & $1.87(11)$ \\
\hline $\mathrm{Ni2}-\mathrm{O} 12$ & $2.10(9)$ & $\mathrm{V} 1-\mathrm{O} 4$ & $1.75(7)$ \\
\hline $\mathrm{Ni} 2-\mathrm{O} 13^{\mathrm{i}}$ & $2.09(8)$ & $\mathrm{V} 1-\mathrm{O} 11$ & $1.64(7)$ \\
\hline $\mathrm{Ni} 2-\mathrm{O} 24$ & $1.85(8)$ & $\mathrm{V} 1-\mathrm{O} 17$ & $1.71(7)$ \\
\hline $\mathrm{Fe} 3-\mathrm{O} 1$ & $1.76(8)$ & $\mathrm{V} 2-\mathrm{O} 2$ & $1.75(11)$ \\
\hline $\mathrm{Fe} 3-\mathrm{O} 2^{\mathrm{iv}}$ & $1.94(9)$ & $\mathrm{V} 2-\mathrm{O} 3$ & $1.73(7)$ \\
\hline $\mathrm{Fe} 3-\mathrm{O}^{\mathrm{iv}}$ & $2.09(8)$ & $\mathrm{V} 2-\mathrm{O} 12$ & $1.92(7)$ \\
\hline $\mathrm{Fe} 3-\mathrm{O}^{\text {iv }}$ & $2.13(7)$ & $\mathrm{V} 2-\mathrm{O} 18$ & $1.50(7)$ \\
\hline $\mathrm{Fe} 3-\mathrm{O} 15$ & $2.12(9)$ & V3-O5 & $1.56(9)$ \\
\hline $\mathrm{Fe} 3-\mathrm{O} 22$ & $2.01(6)$ & V3-O8 & $1.87(7)$ \\
\hline $\mathrm{Ni3}-\mathrm{O} 1$ & $1.76(8)$ & $\mathrm{V} 3-\mathrm{O} 13$ & $1.85(7)$ \\
\hline $\mathrm{Ni} 3-\mathrm{O} 2^{\mathrm{iv}}$ & $1.94(9)$ & $\mathrm{V} 3-\mathrm{O} 15^{\mathrm{ii}}$ & $1.66(8)$ \\
\hline $\mathrm{Ni3}-\mathrm{O}^{\mathrm{iv}}$ & $2.09(8)$ & V4-O6 & $1.8(1)$ \\
\hline $\mathrm{Ni3}-\mathrm{O} 9^{\text {iv }}$ & $2.13(7)$ & V4-O7 & $1.83(8)$ \\
\hline $\mathrm{Ni3}-\mathrm{O} 15$ & $2.12(9)$ & V4-O14 & $1.74(7)$ \\
\hline $\mathrm{Ni3}-\mathrm{O} 22$ & $2.01(6)$ & $\mathrm{V} 4-\mathrm{O} 16^{\mathrm{vi}}$ & $1.59(8)$ \\
\hline $\mathrm{Fe} 4-\mathrm{O}^{\mathrm{v}}$ & $2.27(8)$ & $\mathrm{V} 5-\mathrm{O} 10^{\mathrm{vii}}$ & $1.75(9)$ \\
\hline $\mathrm{Fe} 4-\mathrm{O} 2$ & $2.11(7)$ & $\mathrm{V} 5-\mathrm{O} 19^{\mathrm{ii}}$ & $1.79(7)$ \\
\hline $\mathrm{Fe} 4-\mathrm{O}^{\mathrm{v}}$ & $1.9(1)$ & $\mathrm{V} 5-\mathrm{O} 21$ & $1.77(7)$ \\
\hline $\mathrm{Fe} 4-\mathrm{O} 10^{\mathrm{v}}$ & $2.09(7)$ & $\mathrm{V} 5-\mathrm{O} 23$ & $1.60(7)$ \\
\hline $\mathrm{Fe} 4-\mathrm{O} 16$ & $1.89(9)$ & $\mathrm{V} 6-09^{\text {viii }}$ & $1.73(9)$ \\
\hline $\mathrm{Fe} 4-\mathrm{O} 21$ & $1.85(7)$ & $\mathrm{V} 6-\mathrm{O} 20^{\mathrm{vi}}$ & $1.66(7)$ \\
\hline $\mathrm{Ni4}-\mathrm{O} 1^{\mathrm{V}}$ & $2.27(8)$ & $\mathrm{V} 6-\mathrm{O} 22$ & $1.66(8)$ \\
\hline $\mathrm{Ni4}-\mathrm{O} 2$ & $2.11(7)$ & $\mathrm{V} 6-\mathrm{O} 24$ & $1.83(8)$ \\
\hline
\end{tabular}

в два этапа. На первом этапе определено распределение вероятности квадрупольных расщеплений $\mathrm{P}(\mathrm{QS})$ (рис. $2, b$ ) в экспериментальном спектре. На втором этапе на основе информации, полученной из распре-

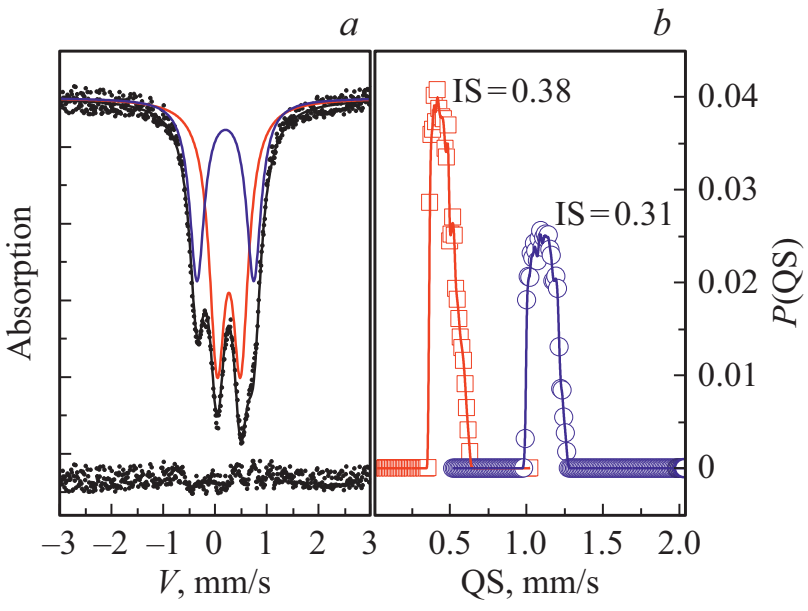

Рис. 2. $a-$ мессбауэровский спектр $\mathrm{NaNiFe}_{2} \mathrm{~V}_{3} \mathrm{O}_{12}$, измеренный при комнатной температуре. Цветными линиями показаны компоненты спектра, параметры которых приведены в табл. 4; $b$ - распределение вероятности квадрупольных расщеплений в экспериментальном спектре.

деления $\mathrm{P}(\mathrm{QS})$, построен модельный спектр, который подогнан к экспериментальному спектру при варьировании всех сверхтонких параметров методом наименьших квадратов в линейном приближении. Результат подгонки сведен в табл. 4.

Распределение вероятности квадрупольных расщеплений в спектре (рис. 2,b) указывает на наличие двух типов позиций атомов железа, имеющих различные величины химических сдвигов и квадрупольных расщеплений. Обращаясь к рентгеновским данным, позиции с параметрами $\mathrm{IS}=0.38 \mathrm{~mm} / \mathrm{s}$ и $\mathrm{QS}=0.45 \mathrm{~mm} / \mathrm{s}$ следует отнести к октаэдрическим кислородным окружениям катионов $\mathrm{Fe}^{3+}(6)$ в $\mathrm{NaNiFe}_{2} \mathrm{~V}_{3} \mathrm{O}_{12}$. Позиции ионов железа в кислородном окружении в виде тригональных пирамид имеют меньшее координационное число и, следовательно, меньшую величину химсдвига, а также значительную степень искажения локального окружения, т.е. большую величину квадрупольного расщепления. Этим условиям отвечают позиции, имеющие мессбауэровские параметры IS $=0.31 \mathrm{~mm} / \mathrm{s}$ и $\mathrm{QS}=1.10 \mathrm{~mm} / \mathrm{s}$ (табл. 4). Таким образом, примерно $60 \%$ атомов железа, входящих в $\mathrm{NaNiFe}_{2} \mathrm{~V}_{3} \mathrm{O}_{12}$, размещаются в октаэдрах

Таблица 4. Мессбауэровские параметры $\mathrm{NaNiFe}_{2} \mathrm{~V}_{3} \mathrm{O}_{12}$. IS - изомерный химический сдвиг относительно $\alpha$-Fe, QS квадрупольное расщепление, $\mathrm{W}$ - ширина линий поглощения, A - площадь под парциальным спектром (долевая заселенность неэквивалентной позиции железом)

\begin{tabular}{c|c|c|c|c}
\hline $\begin{array}{c}\text { IS, мм/c } \\
\pm 0.02\end{array}$ & $\begin{array}{c}\text { QS, } \mathrm{mm} / \mathrm{s} \\
\pm 0.04\end{array}$ & $\begin{array}{c}\mathrm{W}, \mathrm{mm} / \mathrm{s} \\
\pm 0.04\end{array}$ & $\begin{array}{c}\text { A, атом.\% } \\
\pm 0.03\end{array}$ & Позиция \\
\hline 0.38 & 0.45 & 0.37 & 0.59 & $\mathrm{Fe}^{3+}(6)$ \\
0.31 & 1.10 & 0.34 & 0.41 & $\mathrm{Fe}^{3+}(5)$
\end{tabular}




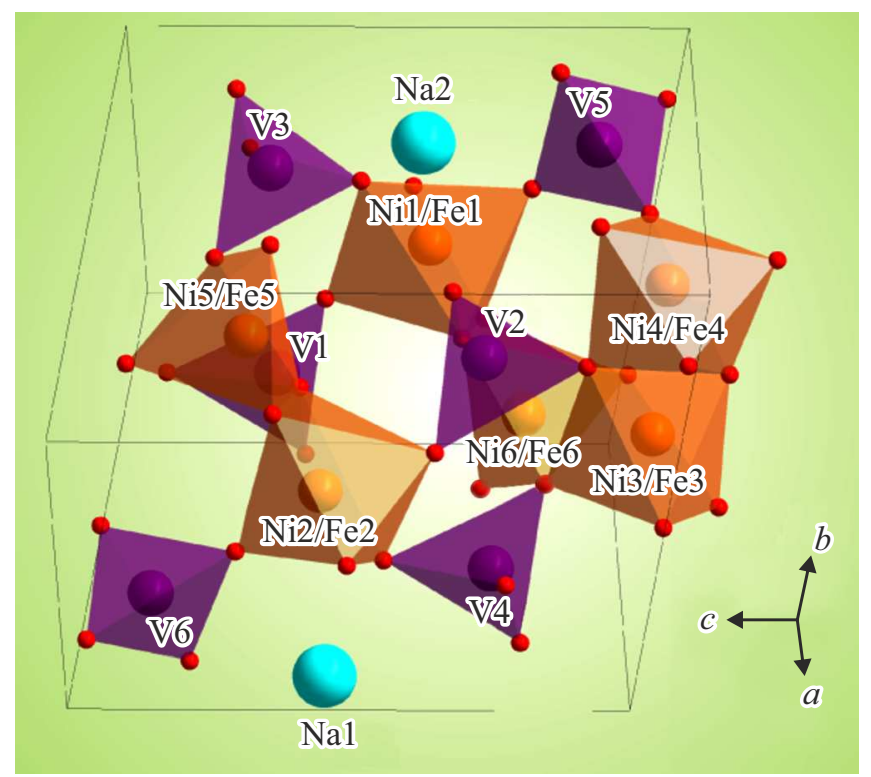

Рис. 3. Кристаллическая структура $\mathrm{NaNiFe}_{2}\left(\mathrm{VO}_{4}\right)_{3}$.

и $\sim 40 \%$ - в тригональных пирамидах. Заселенности позиций железом оценены в предположении равной вероятности эффекта Мёссбауэра для октаэдрических и призматических позиций. Отсутствие гауссовой формы распределений $\mathrm{P}(\mathrm{QS})$, рис. $2, b$, может свидетельствовать в пользу не равновероятной заселенности октаэдрических и призматических позиций железом и, соответственно, никелем.

Таким образом, при уточнении структурных особенностей $\mathrm{NaNiFe}_{2}\left(\mathrm{VO}_{4}\right)_{3}$ методом ядерного гамма-резонанса показано, что катионы железа $\mathrm{Fe}^{3+}$, находятся в высокоспиновом состоянии $\mathrm{S}=5 / 2$ и занимают два типа позиций, отличающихся кислородным окружением: октаэдрическим и в виде тригональных бипирамид. Соответственно, $\sim 80 \%$ никеля размещаются в октаэдрах и $\sim 20 \%-$ в тригональных пирамидах, и формулу ванадата можно представить в следующем виде:

$$
\mathrm{Na}\left[\mathrm{Fe}_{1.2} \mathrm{Ni}_{0.8}\right]\left\{\mathrm{Fe}_{0.8} \mathrm{Ni}_{0.2}\right\}\left(\mathrm{VO}_{4}\right)_{3},
$$

где квадратные скобки содержат октаэдрические, а фигурные скобки - пирамидальные позиции.

В итоге на основании рентгеноструктурных и мёссбауэровских исследований можно считать, что в кристаллической структуре соединения $\mathrm{NaNiFe}_{2}\left(\mathrm{VO}_{4}\right)_{3}$ октаэдры $(\mathrm{Fe} 1 / \mathrm{Ni} 1) \mathrm{O}_{6},(\mathrm{Fe} 2 / \mathrm{Ni} 2) \mathrm{O}_{6},(\mathrm{Fe} 3 / \mathrm{Ni} 3) \mathrm{O}_{6},(\mathrm{Fe} 4 / \mathrm{N} 4) \mathrm{O}_{6}$ и тригональные бипирамиды $(\mathrm{Fe} 5 / \mathrm{Ni5}) \mathrm{O}_{5}, \quad(\mathrm{Fe} 6 / \mathrm{Ni}) \mathrm{O}_{5}$ связаны между собой посредством общих ребер и формируют бесконечные цепи $(. .-(\mathrm{Fe} 1 / \mathrm{Ni} 1)-(\mathrm{Fe} 2 / \mathrm{Ni} 2)-$ $(\mathrm{Fe} 5 / \mathrm{Ni} 5)-(\mathrm{Fe} 4 / \mathrm{Ni} 4)-(\mathrm{Fe} 3 / \mathrm{Ni3})-(\mathrm{Fe} 6 / \mathrm{Ni6})-\ldots)$, тянущиеся вдоль направления а-c. Кроме того, октаэдры и тригональные бипирамиды связаны через вершины с тетраэдрами ванадия $\mathrm{VO}_{4}$. Бесконечные цепи $\ldots-\mathrm{Fe} / \mathrm{Ni}-\mathrm{Fe} / \mathrm{Ni}-\ldots$ сшиваются между собой при помощи тетраэдров $\mathrm{VO}_{4}$ в двумерный слой, который находится в плоскости, заданной двумя векторами а-c и b. В свою очередь, эти слои объединяются между собой образованием $\mathrm{VO}_{4}$, формируя трехмерную структуру - каркас. Кристаллическая структура ванадата $\mathrm{NaNiFe}_{2}\left(\mathrm{VO}_{4}\right)_{3}$ изображена на рис. 3 .

\section{2. Магнитные характеристики $\mathrm{NaNiFe}_{2}\left(\mathrm{VO}_{4}\right)_{3}$}

Магнетизм в поликомпонентной системе $\mathrm{NaNiFe}_{2} \mathrm{~V}_{3} \mathrm{O}_{12}$ обусловлен ионами железа $\mathrm{Fe}^{3+}$ (электронная конфигурация $3 d^{5}$, спин $\mathrm{S}=5 / 2$ ) и никеля $\mathrm{Ni}^{2+}\left(3 d^{8}, \mathrm{~S}=3 / 2\right)$. Ванадий в данном соединении пятивалентен, $\mathrm{V}^{5+}\left(d^{0}\right)$, магнитный момент отсутствует.

На рис. 4-8 приведены результаты экспериментального исследования, характеризующие магнитные свойства $\mathrm{NaNiFe}_{2} \mathrm{~V}_{3} \mathrm{O}_{12}$.

Результаты изучения показали, что полевая зависимость магнитного момента $M(H) \mathrm{NaNiFe}_{2} \mathrm{~V}_{3} \mathrm{O}_{12}$ при комнатной температуре нелинейная и имеет гистерезис (рис. 4). Раскрытие петли магнитного гистерезиса наблюдается в магнитных полях $|H|<2$ kOе. Таким образом, характеристики магнитного поведения образцов $\mathrm{NaNiFe}_{2} \mathrm{~V}_{3} \mathrm{O}_{12}$ указывают на ферромагнитные свойства.

Однако, из приведенных данных также следует, что на кривой полевой зависимости магнитного момента $M(H)$ (рис. 4) наблюдается существенный прирост магнитного момента в магнитных полях выше технического насыщения (область „парапроцесса“). Как известно [14], для многих ферромагнетиков величина намагниченности в области парапроцесса увеличивается незначительно, и ее рост обусловлен изменением ориентации спиновых моментов отдельных электронов, находящихся внутри областей самопроизвольной намагниченности - доменов и оставшихся не повернутыми в направлении поля из-за влияния теплового движения. В исследуемом материале с кристаллической структурой, содержащей шесть различных смешанных позиций магнитных катионов,

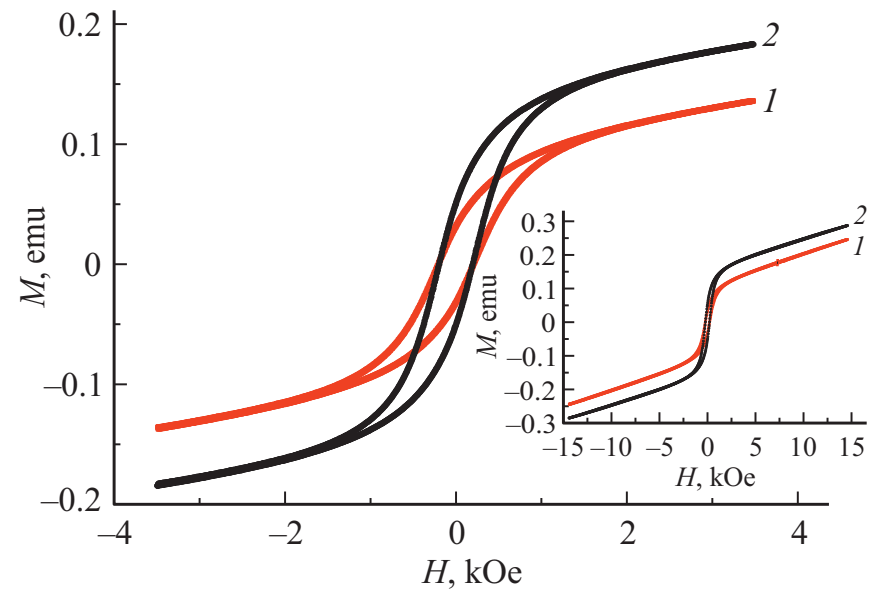

Рис. 4. Петли магнитного гистерезиса в $\mathrm{NaNiFe}_{2} \mathrm{~V}_{3} \mathrm{O}_{12}$ при комнатной температуре. Образцы: массой $m=0.160 \mathrm{~g}(1)$ и $m=0.140 \mathrm{~g}(2)$. На вставке показано поведение магнитного момента в магнитных полях до $20 \mathrm{kOe}$. 
процессы намагничивания, такие как смещение доменных границ, вращение магнитных моментов доменов к направлению приложенного магнитного поля и парапроцесс, можно ожидать, могут частично накладываться друг на друга, приводя к росту магнитного момента в полях выше насыщения. Однако величина прироста намагниченности в области магнитных полей выше $2 \mathrm{kOe}$ велика, и, скорее всего, является следствием проявления парамагнитных свойств исследуемого материала.

На рис. 5 приведены данные измерений полевых зависимостей намагниченности $\mathrm{NaNiFe}_{2} \mathrm{~V}_{3} \mathrm{O}_{12}$ при различных температурах 77, 300 и $830 \mathrm{~K}$. Величина коэрцитивной силы $H_{C}$ при комнатной температуре составляет $\approx 200$ Ое, при переходе к температуре $T=830 \mathrm{~K}$ ее значение снижается в четыре раза. Экспериментальные данные также показывают, что понижение температуры до $77 \mathrm{~K}$ приводит к росту величины магнитного поля, необходимого для размагничивания материала $\mathrm{NaNiFe}_{2} \mathrm{~V}_{3} \mathrm{O}_{12}$. Вероятно, что дефектность образца, влияющая на динамику движения доменов и граничных областей - доменных стенок, является главным фактором, определяющим магнитный гистерезис в $\mathrm{NaNiFe}_{2} \mathrm{~V}_{3} \mathrm{O}_{12}$. Отметим, что при перемагничивании в противоположном направлении значение $H_{C}$ отличается примерно на 10-20 Ое. По-видимому, последняя особенность связана с термомагнитной предысторией образца. На вставке рис. 5 показана зависимость коэрцитивной силы $H_{C}$ от температуры в $\mathrm{NaNiFe}_{2} \mathrm{~V}_{3} \mathrm{O}_{12}$.

Итак, по данным магнитных измерений, с одной стороны, значение $H_{C}$ растет с понижением температуры, что характерно для большинства ферромагнитных материалов. С другой стороны, характер полевых зависимостей статической намагниченности $\mathrm{NaNiFe}_{2}\left(\mathrm{VO}_{4}\right)_{3}$, измеренных при различных температурах, показывает изменение наклона кривых в магнитном поле выше $2 \mathrm{kOe}$ при варьировании температуры (рис. 5), что свойственно для парамагнитного материала.

Анализ кривых намагничивания $\mathrm{NaNiFe}_{2} \mathrm{~V}_{3} \mathrm{O}_{12}$ при температурах $T=300 \mathrm{~K}$ и $T=830 \mathrm{~K}$ (кривая 3), приведенных на рис. 6 , обнаруживает зависимость величины намагниченности от условий проведения эксперимента. При комнатной температуре измерение полевой зависимости намагниченности проводилось дважды: до нагрева образца до температуры $T=830 \mathrm{~K}$ (кривая 2) и после нагрева (кривая 1). Охлаждение образца до комнатной температуры после нагрева до $830 \mathrm{~K}$ приводит к увеличению намагниченности примерно на $40 \%$ в магнитном поле $2 \mathrm{kOe.} \mathrm{Изменение} \mathrm{намагниченности} \mathrm{в} \mathrm{результате}$ термообработки образца сохраняется и в более высоких магнитных полях $(H=10 \mathrm{kOe})$. При этом величина коэрцитивной силы не изменяется.

Таким образом, исследуемый образец, как показывают экспериментальные данные, проявляет как ферромагнитные, так и парамагнитные свойства. Представляют интерес проведение эксперимента, позволяющего исключение влияния парамагнитной составляющей намагниченности на магнитные характеристики образца. С этой

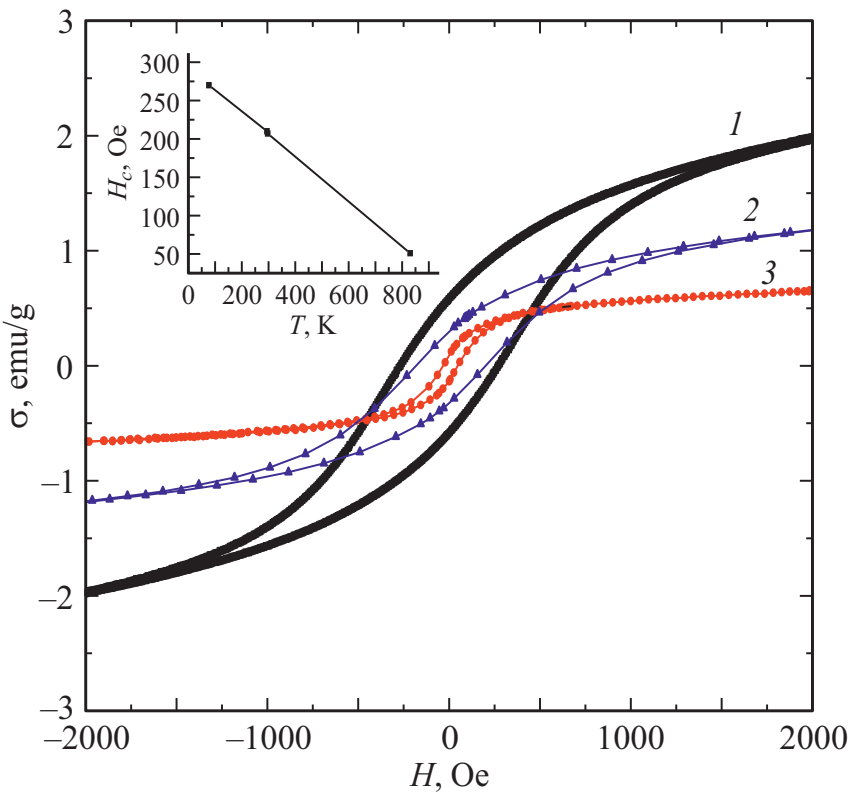

Рис. 5. Магнитополевая зависимость статической намагниченности $\mathrm{NaNiFe}_{2}\left(\mathrm{VO}_{4}\right)_{3}$, измеренная при температурах $T=77 \mathrm{~K}-$ кривая $1,300 \mathrm{~K}-$ кривая $2,830 \mathrm{~K}-$ кривая 3 . На вставке - температурная зависимость коэрцитивной силы $H_{C}$.

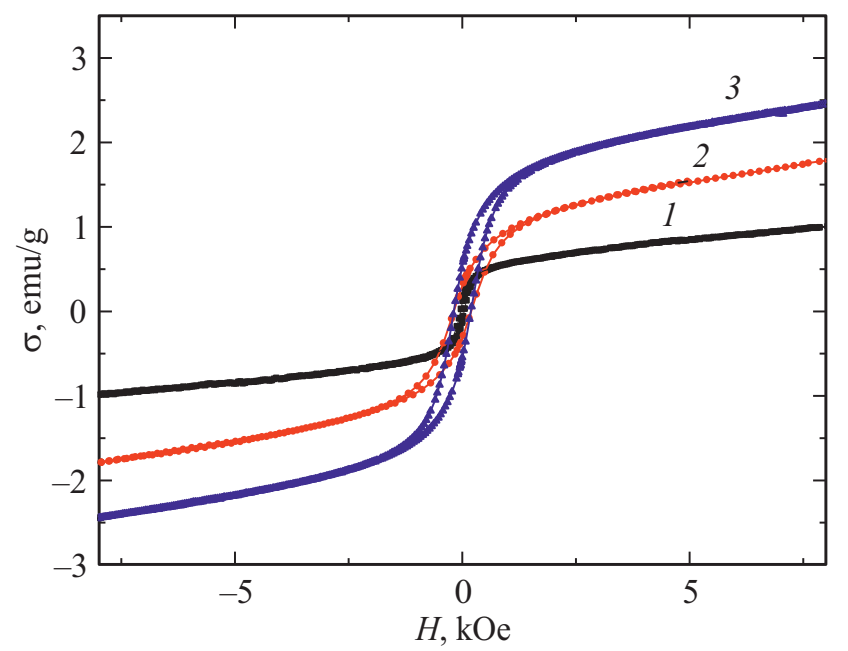

Рис. 6. Зависимость магнитных характеристик образца $\mathrm{NaNiFe}_{2} \mathrm{~V}_{3} \mathrm{O}_{12}$ от термомагнитной предыстории: петли магнитного гистерезиса при температурах $830 \mathrm{~K}$ (кривая 1) и $300 \mathrm{~K}$ (кривая 2 - измерение проведено до нагрева образца, кривая 3 - после нагрева образца до температуры $830 \mathrm{~K}$.

целью была измерена температурная зависимость остаточной намагниченности в диапазоне температур от гелиевой до комнатной. На рис. 7 представлена кривая температурной зависимости остаточной намагниченности $\mathrm{NaNiFe}_{2} \mathrm{~V}_{3} \mathrm{O}_{12}$, измеренная на СКВИД-магнитометре в отсутствии внешнего магнитного поля $H$. Результаты измерения показали, что форма кривой (рис. 7) характерна для магнетика с ферромагнитным типом упо- 


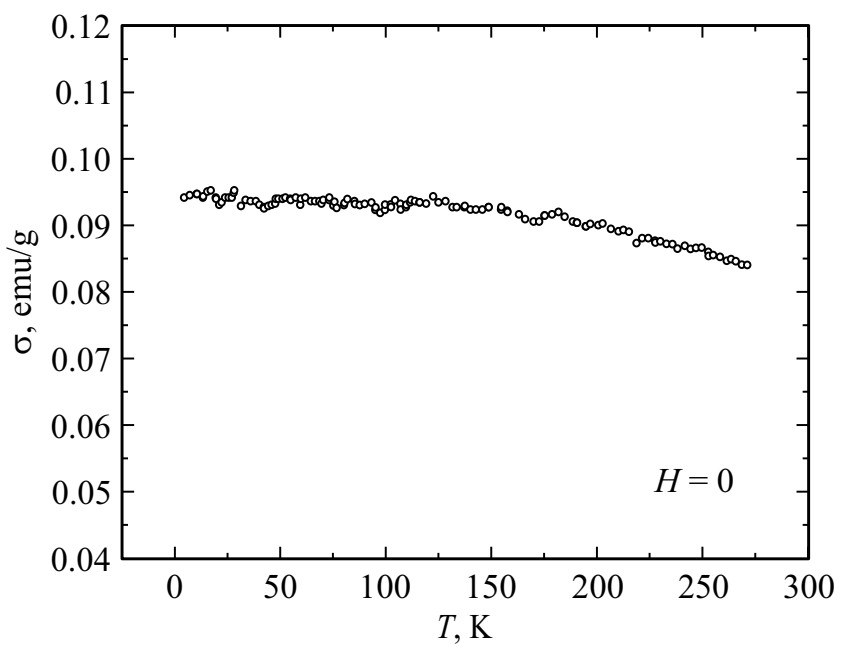

Рис. 7. Температурная зависимость остаточной намагниченности $\mathrm{NaNiFe}_{2}\left(\mathrm{VO}_{4}\right)_{3}$. Образец массой $m=0.120 \mathrm{~g}$.

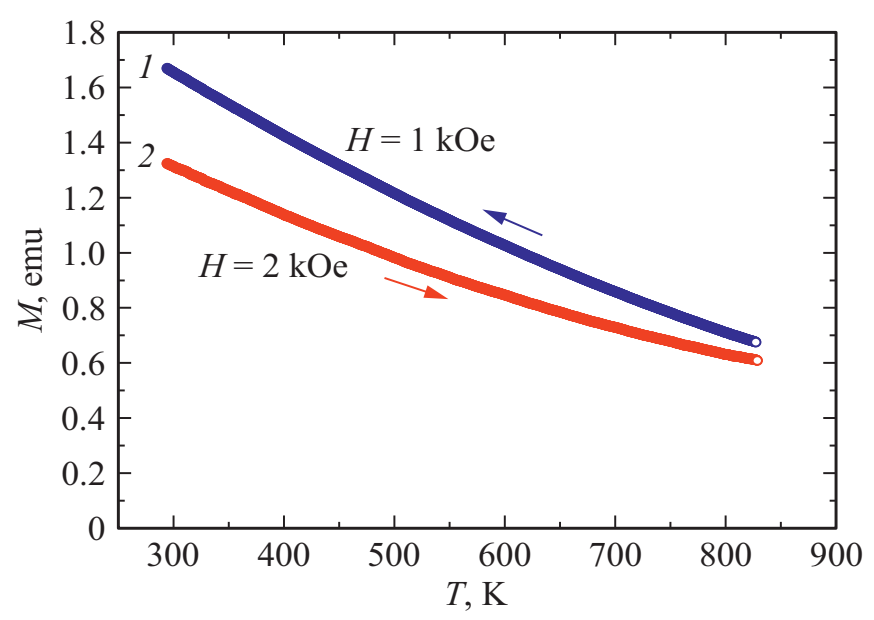

Рис. 8. Температурные зависимости магнитного момента $\mathrm{NaNiFe}_{2}\left(\mathrm{VO}_{4}\right)_{3}$, измеренные в магнитных полях $H=1 \mathrm{kOe}$ и $H=2 \mathrm{kOе}$ в интервале температур $300-830 \mathrm{~K}$ в режимах охлаждения (кривая 1) и нагрева (кривая 2) образца.

рядочения, самопроизвольная намагниченность образца $\sigma_{T=0}=0.095 \mathrm{emu} / \mathrm{g}(T=0 \mathrm{~K})$.

Отметим также особенности поведения соединения $\mathrm{NaNiFe}_{2} \mathrm{~V}_{3} \mathrm{O}_{12}$, связанные с его термомагнитной предысторией. На рис. 8 приведены кривые температурной зависимости магнитного момента, измеренные в магнитном поле $H=1000,2000$ Ое в интервале температур $300-830 \mathrm{~K}$ в режимах охлаждения и нагрева образца $\mathrm{NaNiFe}_{2} \mathrm{~V}_{3} \mathrm{O}_{12}$. Опытные данные свидетельствуют о том, что магнитный момент образца при комнатной температуре увеличивается примерно на $27 \%$ после нагрева от 300 до $830 \mathrm{~K}$, несмотря на различие величин приложенного магнитного поля.

При интерпретации свойств ванадата $\mathrm{NaNiFe}_{2}\left(\mathrm{VO}_{4}\right)_{3}$ со сложными составом и кристаллографической структурой возникает ряд трудностей, обусловленных наличием шести неэквивалентных кристаллографических позиций $\mathrm{Fe} i / \mathrm{Ni} i(i=1-6)$, присутствием в них разновалентных катионов, заселенных неравномерно и приводящих к неоднородности структуры. Кроме того, при внедрении ионов никеля в позиции ионов железа из-за различия их ионных радиусов $r\left(r_{\mathrm{Fe}^{3+}}=0.67 \AA\right.$ и $\left.r_{\mathrm{Ni}^{2+}}=0.78 \AA\right)$ возникают искажения кристаллической решетки.

Отметим, что при синтезе образцов из смесей порошкообразных оксидов с помощью твердофазной реакции может происходить образование побочных продуктов, в том числе магнитных. Трудности в расшифровке опытных данных, связанные с присутствием примеси, требуют анализа их возможного влияния на магнитные свойства $\mathrm{NaNiFe}_{2}\left(\mathrm{VO}_{4}\right)_{3}$. Одной из вероятных примесей может быть гематит $\alpha-\mathrm{Fe}_{2} \mathrm{O}_{3}$. Известно [15-17], что гематит является ромбоэдрическим антиферромагнетиком с температурой Нееля $T_{\mathrm{N}}=950 \mathrm{~K}$, в окрестности температуры $T_{\mathrm{M}} \approx 260 \mathrm{~K}$ (температура Морина) происходит переориентация спинов с поворотом на $90^{\circ}$, при этом устанавливается неколлинеарное упорядочение спинов (со скосом друг относительно друга на $5^{\circ}$ ), приводящее к слабому магнетизму. Анализируя возможное влияние примеси $\alpha-\mathrm{Fe}_{2} \mathrm{O}_{3}$ на магнитные свойства исследуемых образцов $\mathrm{NaNiFe}_{2}\left(\mathrm{VO}_{4}\right)_{3}$, отметим, что не обнаружены аномалии магнитных свойств в окрестности температуры $T_{\mathrm{M}} \approx 260 \mathrm{~K}$, соответствующей спинпереориентационному переходу Морина между коллинеарным антиферромагнитным и слабоферромагнитным состояниями. К возможным примесям, не обнаруженным в ходе проведенных экспериментов, можно отнести магнетит $\mathrm{Fe}_{3} \mathrm{O}_{4}$ - соединение со структурой обращенной шпинели $\mathrm{Fe}^{3+}\left[\mathrm{Fe}^{2+} \mathrm{Fe}^{3}\right] \mathrm{O}_{4}$. При комнатной температуре феррит $\mathrm{Fe}_{3} \mathrm{O}_{4}$ является ферримагнетиком, разрушение ферримагнитного упорядочения тепловым движением происходит при температуре 850-860 K, коэрцитивная сила $H_{c}=2.1 \mathrm{Oe}[18,19]$. При температуре $T=100-120 \mathrm{~K}$ (температура Вервея) имеет место низкотемпературное структурное превращение магнетита, проявляемое на температурных зависимостях намагниченности, теплоемкости, удельного сопротивления $[18,19]$. В образце $\mathrm{NaNiFe}_{2}\left(\mathrm{VO}_{4}\right)_{3}$ не обнаружена аномалия на температурной зависимости магнитного момента $M(T)$ при $T=100-120 \mathrm{~K}$. Кроме того, отметим, что в исследуемых образцах согласно данным мессбауэровских исследований отсутствуют катионы $\mathrm{Fe}^{2+}$. В качестве побочных продуктов твердотельной реакции следует рассмотреть никелевый феррит $\mathrm{NiFe}_{2} \mathrm{O}_{4}$, а также оксид никеля $\mathrm{NiO}$. Соединение $\mathrm{NiFe}_{2} \mathrm{O}_{4}$ имеет кристаллическую структуру обращенной шпинели, температуру Кюри $T_{c}=863 \mathrm{~K}$, коэрцитивную силу $H_{c}=5$ Ое. Кубический кристалл $\mathrm{NiO}$ - антиферромагнетик, температура Нееля $T_{\mathrm{N}}=793 \mathrm{~K}$ [18]. Таким образом, анализ свойств вероятных примесей показывает, что магнетизм синтезированного соединения $\mathrm{NaNiFe}_{2}\left(\mathrm{VO}_{4}\right)_{3}$ имеет характеристики, отличительные от таковых возможных побочных продуктов твердотельной реакции. 
Кроме того, проведены измерения теплоемкости методом адиабатического калориметра в режиме непрерывного нагрева в области температур 77-870 К. Отметим в данном температурном диапазоне отсутствие особенностей в поведении температурной зависимости теплоемкости, свидетельствующих о наличии фазовых превращений в образце.

Итак, основными особенностями синтезированного образца $\mathrm{NaNiFe}_{2} \mathrm{~V}_{3} \mathrm{O}_{12}$, характеризующего его магнитные свойства, является наличие магнитного момента, зависящего от температуры (рис. 5,7), магнитного поля (рис. 4,5) и его термомагнитной предыстории (рис. 6,8). Анализ поведения магнитных характеристик $\mathrm{NaNiFe}_{2}\left(\mathrm{VO}_{4}\right)_{3}$ показывает, что материал наряду c ферромагнитными свойствами проявляет свойства парамагнетика. Возможным объяснением совокупности экспериментальных фактов может быть предположение о том, что образец представляет собой парамагнитную матрицу с ферромагнитными включениями - кластерами. Последние определяют ферромагнитное поведение образца. Увеличение намагниченности после „отжига“ в магнитном поле в данной концепции свидетельствует, по-видимому, не только о выстраивании отдельных магнитных частиц материала вдоль направления приложенного магнитного поля, увеличении их объема, но и об образовании новых ферромагнитных включений. Отметим также, что с повышением температуры наблюдается снижение остаточной намагниченности. Однако температура Кюри ферромагнитных образований, видимо, существенно превышает максимально достигнутую в эксперименте температуру $830 \mathrm{~K}$. Можно предположить, что взаимодействие между ионами железа и никеля, занимающими неодинаковые позиции в кристаллической решетке $\mathrm{NaNiFe}_{2}\left(\mathrm{VO}_{4}\right)_{3}$, приводит к образованию неоднородной системы ферромагнитных кластеров, свойства которых могут зависеть, в частности, от межионных расстояний железо-железо, никель-никель, железо-никель. Упорядочение кластеров, природа которых не ясна и требует проведения дополнительных исследований, оказывает существенное влияние на формирование магнитных свойств изучаемого материала в широком диапазоне температур.

\section{4. Заключение}

Новый оксид ванадия $\mathrm{NaNiFe}_{2}\left(\mathrm{VO}_{4}\right)_{3}$ получен твердофазным синтезом и исследован методами рентгеновской дифракции, гамма-резонанса и магнитометрии. На основании вышеизложенного можно сделать следующие выводы.

Установлено, что образцы имеют триклинную кристаллическую структуру с пространственной группой $P 1$ и параметрами решетки $a=6.7559(1) \AA$, $b=8.1309(2) \AA, \quad c=9.8098(2) \AA, \quad \alpha=106.346(2)^{\circ}$, $\beta=104.300(2)^{\circ}, \gamma=102.143(2)^{\circ}, V=477.83$ (2) $\AA^{3}$. В структуре $\mathrm{NaNiFe}_{2}\left(\mathrm{VO}_{4}\right)_{3}$ имеется шесть неэквива- лентных смешанных позиций с локализацией катионов разных сорта и валентности $\left(\mathrm{Fe}^{3+} / \mathrm{Ni}^{2+}\right)$ в одной и той же позиции.

Ионы железа, входящего в $\mathrm{NaNiFe}_{2} \mathrm{~V}_{3} \mathrm{O}_{12}$, находятся в трехвалентном высокоспиновом состоянии и занимают октаэдрические $(\sim 60 \%)$ и пирамидальные $(\sim 40 \%)$ позиции с неравновероятной заселенностью их атомами железа и никеля. Таким образом, формула соединения имеет вид $\mathrm{Na}\left[\mathrm{Fe}_{1.2} \mathrm{Ni}_{0.8}\right]\left\{\mathrm{Fe}_{0.8} \mathrm{Ni}_{0.2}\right\}\left(\mathrm{VO}_{4}\right)$, где квадратные скобки содержат октаэдрические, а фигурные скобки пирамидальные позиции.

Анализ магнитных свойств $\mathrm{NaNiFe}_{2}\left(\mathrm{VO}_{4}\right)_{3}$ показывает, что как температурные, так и полевые зависимости намагниченности демонстрируют суперпозицию пара- и ферромагнитного поведения. Физическая природа наблюдаемых особенностей магнетизма $\mathrm{NaNiFe}_{2}\left(\mathrm{VO}_{4}\right)_{3}$, возможно, связана с магнитным взаимодействием между ионами железа и никеля с различными и не равномерно заселенными позициями в кристаллической решетке, способствующем образованию магнитных кластеров, упорядочение которых приводит к ферромагнетизму.

\section{Благодарности}

Авторы выражают благодарность В.С. Бондареву за проведение исследований тепловых свойств $\mathrm{NaNiFe}_{2}\left(\mathrm{VO}_{4}\right)_{3}$.

\section{Конфликт интересов}

Авторы заявляют, что у них нет конфликта интересов.

\section{Список литературы}

[1] M.A. Lafontaine, J.M. Grenéche, Y. Laligant, G. Férey. J. Solid State Chem. 108, 1 (1994).

[2] Т.В. Дрокина, Г.А. Петраковский, О.А. Баюков, М.С. Молокеев, А.М. Воротынов, С.И. Попков, Д.А. Великанов. ФТТ 62, 247 (2020).

[3] N. Guskos, G. Zolnierkiewicz, J. Typek, R. Szymczak, A. Guskos, P. Berczynski, A. Blonska-Tabero. Mater. Sci. Poland 31, 601(2013).

[4] Т.В. Дрокина, Г.А. Петраковский, О.А. Баюков, А.М. Воротынов, Д.А. Великанов, М.С. Молокеев. ФТТ 58, 1913 (2016).

[5] G. Zolnierkiewicz, N. Guskos, J. Typek, E.A. Anagnostakis, A. Blonska-Tabero, M. Bosacka. J. Alloys Compd. 471, 28 (2009).

[6] J.M. Hughes, J.W. Drexler, C.F. Campana, M.L. Malinconico. Am. Mineral. 73, 181 (1988).

[7] A.A. Belik. Mater. Res. Bull. 34, 12, 1973 (1999).

[8] A.V. Koshelev, K.V. Zakharov, L.V. Shvanskaya, A.A. Shakin, D.A. Chareev, S. Kamusella, H.-H. Klauss, K. Molla, B. Rahaman, T. Saha-Dasgupta, A.P. Pyatakov, O.S. Volkova, A.N. Vasiliev. Phys. Rev. Appl. 10, 034008 (2018).

[9] Т.В. Дрокина, Г.А. Петраковский, А.Л. Фрейдман, М.С. Молокеев, Е.Г. Резина. Магнитодиэлектрический оксидный керамический материал. Патент РФ № 2592867 от 27.07.2016. 
[10] Bruker AXS TOPAS V4: General profile and structure analysis software for powder diffraction data. User s Manual. Bruker AXS, Karlsruhe, Germany (2008).

[11] Д.А. Великанов. Вестн. СибГАУ 2, 48, 176 (2013).

[12] Д.А. Великанов. Вибрационный магнитометр. Патент РФ № 2341810 от 20.12.2008.

[13] F.D. Martin, H. Müller-Buschbaum. Z. Naturforsch. B 50, 1, $51(1995)$

[14] К.П. Белов. Упругие, тепловые и электрические явления в ферромагнетиках. Гос. изд-во техн.-теор. лит., М. (1957). $279 \mathrm{c}$.

[15] L. Nèel, R. Pauthenet. C.R. Acad. Sci. Paris 234, 2172 (1952).

[16] L. Nèel. Rev. Mod. Phys. 25, 58 (1953).

[17] J.O. Artman, J.C. Murphy, S. Foner. Phys. Rev. 138, A912 (1965).

[18] Итоги науки. Вып. 4. Антиферромагнетизм и ферриты. АН CCCP, M. (1962). 215 c.

[19] К.П. Белов. УФН 163, 5, 53 (1993).

Редактор Ю.Э. Китаев 